\title{
Computed tomographically guided transthoracic biopsy of a cardiac mass in the right ventricular outflow tract
}

\author{
Brian J. Burkett, MD, ${ }^{\mathrm{a}}$ Arman Arghami, MD, ${ }^{\mathrm{b}}$ Melanie C. Bois, MD, ${ }^{\mathrm{c}}$ Jerome F. Breen, MD, ${ }^{\mathrm{a}}$ and \\ Jason R. Young, MD, ${ }^{\text {a }}$ Rochester, Minn
}

\author{
From the Departments of ${ }^{\mathrm{a}}$ Radiology, ${ }^{\mathrm{b}}$ Cardiovascular Surgery, and ${ }^{\mathrm{c}}$ Lab Medicine and Pathology, Mayo Clinic, \\ Rochester, Minn. \\ No funding was provided for this article. \\ Disclosures: Authors have nothing to disclose with regard to commercial support. \\ Received for publication April 10, 2019; revisions received May 23, 2019; accepted for publication May 27, 2019; \\ available ahead of print June 27, 2019. \\ Address for reprints: Jason R. Young, MD, Department of Radiology, Mayo Clinic, 200 First St SW, Rochester, \\ MN 55905 (E-mail: young.jason@ mayo.edu). \\ J Thorac Cardiovasc Surg 2020;159:e115-8 \\ $0022-5223 / \$ 36.00$ \\ Copyright (C) 2019 by The American Association for Thoracic Surgery \\ https://doi.org/10.1016/j.jtcvs.2019.05.053
}

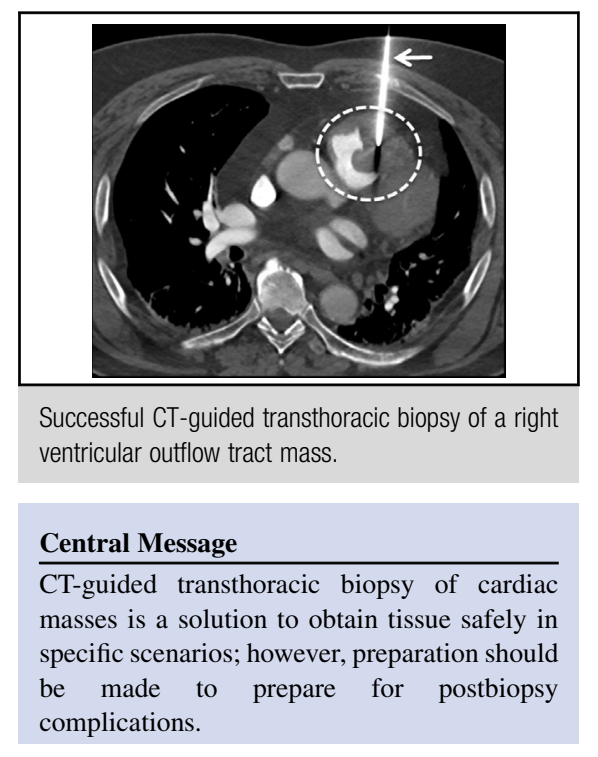

See Commentary on page e119.
Obtaining a tissue diagnosis for unresectable intracardiac tumors can be a dilemma. Although open cardiac biopsy is often technically feasible, it may delay the start of appropriate systemic treatment. Although endoluminal catheterdirected biopsy is helpful in many cases, it may not produce diagnostic samples; especially in tumors with reactive surrounding capsules. We present an infrequently described, image-guided biopsy technique adapted to sample heart masses that can minimize morbidity and allow the timely initiation of systemic therapy.

\section{CLINICAL SCENARIO}

A 73-year-old man presented with a right ventricular mass discovered on a routine surveillance computed tomographic (CT) study for moderately differentiated pT3b hepatocellular carcinoma (HCC) after definitive resection through a right partial hepatectomy 3 years previously without chemotherapy or radiation therapy. The patient was free of symptoms and otherwise had no medical problems. He was initially treated with enoxaparin for a presumed right ventricular thrombus. The mass did not resolve according to serial echocardiograms, however, which showed normal left and right ventricular systolic ejection fractions, no valvular disease, and mildly elevated velocities around the mass. Subsequently, cardiac magnetic resonance imaging (Figure 1) demonstrated an enhancing mass infiltrating the anterior right ventricle and right ventricular outflow tract (RVOT). Primary considerations were metastatic HCC, angiosarcoma, and lymphoma.
Video clip is available online.

Serum liver function tests and $\alpha$-fetoprotein were normal. An abdominal magnetic resonance imaging study yielded negative results for recurrent $\mathrm{HCC}$, and a fludeoxyglucose F 18 (INN fludeoxyglucose $\left[{ }^{18} \mathrm{~F}\right]$ ) positron emission tomographic and CT study only showed hypermetabolism in the right heart mass, without lesions of concern for metastasis throughout the torso. Endomyocardial biopsy of the mass through a right internal jugular vein catheterization with a $7 \mathrm{~F}$ sheath, transthoracic ultrasound guidance, and a $50-\mathrm{cm}$ bioptome device only showed interstitial fibrosis within 6 samples (Figure 2, C). A multidisciplinary discussion among representatives from cardiology, oncology, cardiac surgery, and radiology ultimately resulted in the decision to pursue a CT-guided percutaneous transthoracic biopsy. The decision was based on several factors, including probable fibrous capsule of the mass, which precluded a catheter biopsy approach; the unresectability of the mass; and the mass location, along with the delay in initiation of chemotherapy and morbidity associated with an open surgical biopsy. The coronary vessels were mapped before the procedure by means of image review with an expert in cardiac imaging.

Within the interventional CT suite, the patient was placed under general anesthesia, with extracorporeal 


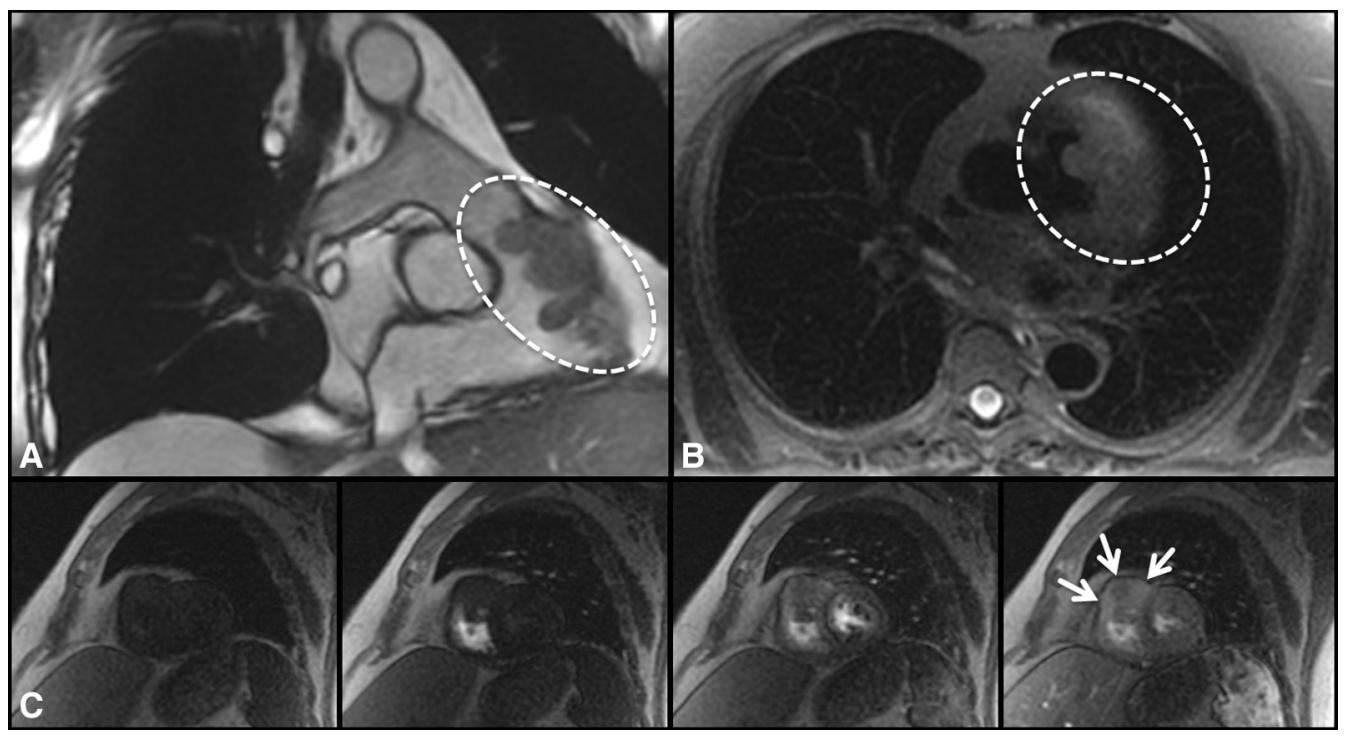

FIGURE 1. Cardiac magnetic resonance images of a 73-year-old man with a history of hepatocellular carcinoma in remission for 3 years after definitive hepatectomy. A, Fast imaging employing steady state acquisition (FIESTA) of the right ventricular inflow tract plane demonstrates an infiltrative, multilobulated mass extending along the anterolateral right ventricular outflow tract. B, Axial triple inversion recovery demonstrates mild edema throughout the mass. C, Short-axis sequential vascular model perfusion shows mild enhancement throughout the mass (arrows), which excludes thrombus. The combination of these findings and the absence of a mass on previous surveillance imaging points to an aggressive tumor, such as metastatic disease or soft-tissue sarcoma. The absence of pericardial effusion makes soft-tissue sarcoma less likely.

membrane oxygenation on standby. A cardiac surgeon and expert in ultrasound placement of pericardial drains were on standby. The pathology team was present for rapid on-site cytologic evaluation (Figure 2, D). Patient respirations had a significant impact on shifting the RVOT mass, so manipulation of the needle and firing the biopsy were performed on ventilator end-expiration. A 17/18-gauge introducer needle was advanced into the RVOT component of the mass with the guidance of intermittent stationary CT images of the mass, and the location was confirmed with pulmonary embolism protocol enhanced CT (Figure 2, A). A total of 3 biopsy samples, each 18 gauge and $2.0 \mathrm{~cm}$ long, were obtained with a spring-loaded core biopsy device. Care was taken to avoid penetrating the endocardium with the biopsies by aligning the introducer needle within the central aspect of the mass, measuring biopsy length before biopsy, and allowing a plane of soft tissue between the biopsy tip and luminal margin of mass. Pathology confirmed metastatic HCC (Figure 2, E and $F$ ). The introducer needle was then removed on ventilator end-expiration. Ten minutes after the biopsy, a repeat CT excluded postbiopsy complications (Figure 2, B). An annotated step-by-step video of the procedure is provided (Video 1). No complications were evident on a postbiopsy transthoracic echocardiogram performed in the CT suite. The patient was free of symptoms after recovering from anesthesia. He was admitted overnight for observation and was discharged the next day after an uneventful evening.

\section{DISCUSSION}

To our knowledge, we present here the first case of CTguided RVOT mass biopsy. Transluminal catheter directed endomyocardial approaches with either fluoroscopic or ultrasound guidance are well-established biopsy techniques. ${ }^{1}$ Alternatives, however, include ultrasound- and CT-guided percutaneous transthoracic approaches, which may be valuable in select cases. ${ }^{2-5}$

In this case, CT-guided transthoracic biopsy proved safe and successful after catheter-directed endomyocardial biopsy had been ineffective in diagnosing isolated HCC metastasis to the right ventricle and RVOT, which is an incredibly rare lesion. ${ }^{6}$ The clinical presentation of the cardiac metastasis in our case mirrors descriptions in the literature: symptom-free patients who had undergone partial hepatectomy for HCC with no hepatic recurrence. Those patients were treated with chemotherapy, and with surgical resection in 1 case. $^{6}$

A $\beta$-blocker was not used in our case, because introducer needle manipulation could not be coordinated with the cardiac cycle. Neither transesophageal nor transthoracic echocardiography was utilized because of the suboptimal insonification angle of the biopsy needle; the use of a spring-loaded biopsy device, precluding control of biopsy depth with ultrasound; and the desire to minimize time with introducer needle in the cardiac mass between modalities. Concurrent use of ultrasound, however, may be helpful in different clinical scenarios. The use of a hybrid operating room with $\mathrm{C}$-arm fluoroscopic cone-beam CT 


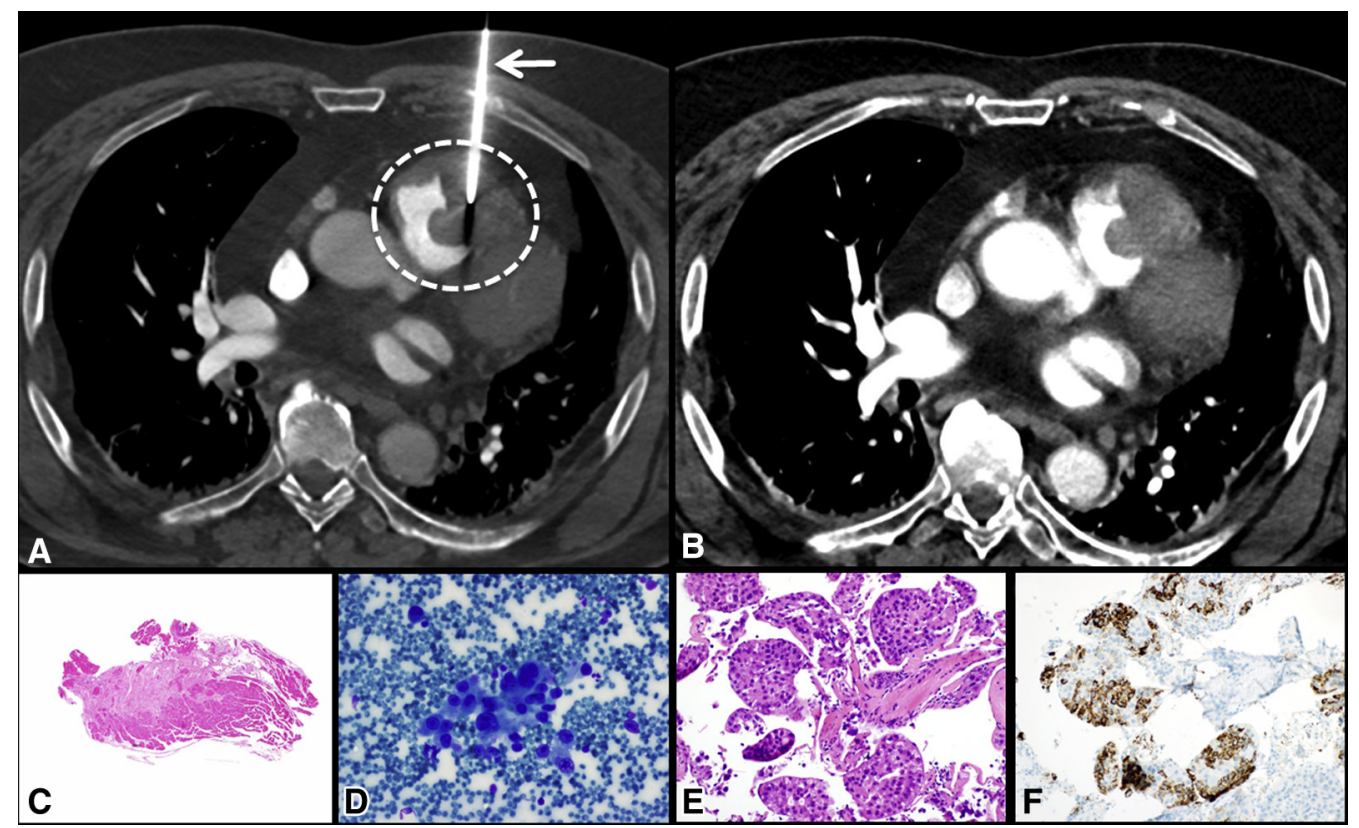

FIGURE 2. Computed tomographically guided transthoracic biopsy of an aggressive right ventricular outflow tract mass after nondiagnostic endovascular bioptome biopsy in a 73-year-old man with a history of hepatocellular carcinoma. A window between the sternum and the left ribs allowed the introduction of an introducer needle into the right ventricular outflow tract mass. A, Computed tomographic angiography pulmonary embolism protocol confirm the targeted position of the introducer needle within the mass. The needle was slightly withdrawn before 3 core biopsy samples $(18$ gauge and $2.0 \mathrm{~cm}$ long $)$ were obtained. B, A repeat computed tomographic angiogram 10 minutes after removal of the introducer needle excludes early complications. C, Endomyocardial biopsy from the previous endovascular approach showing a nondiagnostic sample with nonspecific cardiac hypertrophy and fibrosis (hematoxylin and eosin, $40 \times$ original magnification). D and E, On-site evaluation of the computed tomographically guided transthoracic core biopsy demonstrates loosely cohesive clusters of malignant cells with prominent nucleoli and anisonucleosis ( $\mathrm{D}$; air-dried cytologic preparation, $400 \times$ original magnification), malignant cells with a nodular growth pattern and eosinophilic cytoplasm (E; hematoxylin and eosin stain, $200 \times$ original magnification), and immunohistochemical confirmation of hepatocellular primary tumor (F; hepatocyte immunohistochemistry, 200× original magnification).

was considered and decided against, in response to worries about reaching prohibitive levels of patient radiation exposure and poor image quality relative to standard CT. Cardiac pacing pads were not preemptively placed in this case, because the mass was distant from the primary conduction system; however, such placement should be considered. Our patient was admitted overnight for observation with telemetry because of unknown potential complications; however, this observation period may be overly cautious.

A transthoracic CT-guided approach may offer several advantages relative to catheter-directed jaw-type bioptome approaches, including the absence of the need for vascular access; a larger sample size; the sampling of tissue from the central portion of the mass rather than the endocardial aspect, which may be fibrotic; the large anatomic field of view with options to cross lung and bone (particularly the sternum) if needed; precise control of the biopsy needle; and the ability to visualize complications globally immediately after the biopsy. In addition, samples from CT biopsy are amenable to rapid on-site cytologic evaluation, which is challenging with endomyocardial biopsies because the

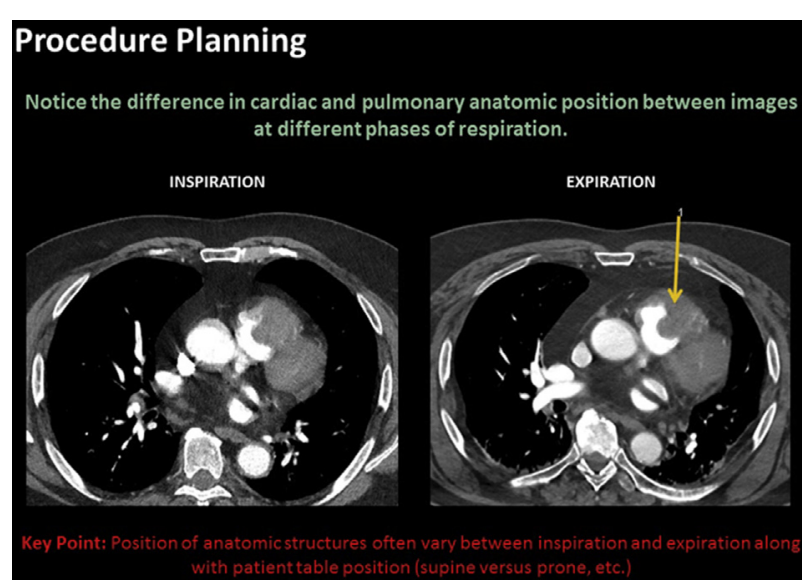

VIDEO 1. A slide show details the computed tomographically (CT) guided cardiac biopsy technique step by step, from the procedure planning to the removal of the introducer needle. ECG, Electrocardiography, ECMO, extracorporeal membrane oxygenation; $M R I$, magnetic resonance imaging; RVOT, right ventricular outflow tract. Video available at: https://www.jtcvs. org/article/S0022-5223(19)31156-0/fulltext. 
myocytes do not tend to exfoliate onto slides. Endoluminal approaches are well established, however, and have the advantages of postbiopsy bleeding back into the blood pool and an approach that avoids crossing vital structures, such as epicardial arteries. Furthermore, new endoluminal techniques with a core biopsy system (traditionally used for transvenous liver biopsies) and transthoracic or intravascular ultrasound guidance are being described, with histopathologic diagnosis achieved in $72 \%$ of cases and the most common complication $(7 \%)$ being ventricular arrhythmias requiring cardioversion. ${ }^{7,8}$

Although CT-guided transthoracic biopsy of RVOT cardiac masses is novel, this case illustrates that it is feasible. Masses in the anterior pericardium and right and left atria (when large) may also be optimal targets for a transthoracic CT biopsy. Potential complications of CT-guided transthoracic biopsy include pneumothorax, hemopericardium, air embolism, induction of cardiac arrhythmias, and vascular injury, particularly to the internal thoracic and epicardial arteries. Percutaneous transthoracic biopsy is believed to carry a greater risk of hemorrhage than endovascular biopsy, and preparations must be made to address hemopericardium as an emergency. ${ }^{2}$

Although transluminal catheter-directed endomyocardial biopsies are currently the standard of care, CT-guided approaches should be considered and may be the optimal technique for large, lobulated cardiac masses that would be biopsied without penetrating the endocardium. ${ }^{2}$ This minimally invasive technique allowed our patient to proceed with chemotherapy 2 days after his biopsy. More data are needed regarding CT-guided approaches, however, and every case should be carefully scrutinized, with extra attention to epicardial arteries, cardiac valves, and expected location of the conduction system along the biopsy needle path.

\section{References}

1. From AM, Maleszewski JJ, Rihal CS. Current status of endomyocardial biopsy Mayo Clin Proc. 2011;86:1095-102.

2. Daliri A, Oehring K, Moosdorf RG, Franke FE, Kalinowski M, Zahedi F, et al. Percutaneous left atrial cardiac biopsy with CT fluoroscopy guidance. J Vasc Interv Radiol. 2007;18:909-13.

3. Gorospe L, Cabañero-Sánchez A, Muñoz-Molina GM, Ayala-Carbonero AM, Fernández-Méndez MA. Computed tomography-guided core needle biopsy of cardiac angiosarcoma. Arq Bras Cardiol. 2018;110:493-4. Portuguese.

4. Nakata A, Hirota S, Takazukura E. Primary cardiac lymphoma diagnosed by percutaneous needle biopsy. Int J Cardiol. 1998;65:201-3.

5. Yamagami T, Kato T, Tanaka O, Hirota T, Ito K, Nishimura M, et al. Percutaneous needle biopsy under CT fluoroscopic guidance for cardiac tumor during continuous intravenous injection of contrast material. J Vasc Interv Radiol. 2005;16: 559-61.

6. Kim SB, Shin YC, Kwon SU. Isolated metastasis of hepatocellular carcinoma in the right ventricle: a case report. Medicine (Baltimore). 2016;95:e5544.

7. Sze DY, Lee DP, Hofmann LV, Petersen B. Biopsy of cardiac masses using a stabilized intracardiac echocardiography-guided system. J Vasc Interv Radiol. 2008; 19:1662-7.

8. Reddy G, Maor E, Bois MC, Chandrasekaran K, Rihal CS, Nishimura RA, et al. Percutaneous transcatheter biopsy for intracardiac mass diagnosis. EuroIntervention. 2017;13:e1436-43. 